Review Article

\title{
Exosomes as Mediators of Cell-to-Cell Communication in Thyroid Disease
}

\author{
Yi Wang, ${ }^{1}$ Feng Xu, ${ }^{1}$ Jia-Yu Zhong, ${ }^{1}$ Xiao Lin ${ }^{D},{ }^{2}$ Su-Kang Shan, ${ }^{1}$ Bei Guo, ${ }^{1}$ \\ Ming-Hui Zheng, ${ }^{1}$ and Ling-Qing Yuan (i) ${ }^{1}$ \\ ${ }^{1}$ Department of Metabolism and Endocrinology, National Clinical Research Center for Metabolic Diseases, \\ Hunan Provincial Key Laboratory of Metabolic Bone Diseases, The Second Xiang-Ya Hospital, Central South University, \\ Changsha, Hunan, China \\ ${ }^{2}$ Department of Radiology, The Second Xiang-Ya Hospital, Central South University, Changsha, Hunan, China
}

Correspondence should be addressed to Ling-Qing Yuan; allenylq@hotmail.com

Received 29 December 2019; Revised 21 February 2020; Accepted 28 February 2020; Published 28 April 2020

Academic Editor: Claudio Casella

Copyright ( 2020 Yi Wang et al. This is an open access article distributed under the Creative Commons Attribution License, which permits unrestricted use, distribution, and reproduction in any medium, provided the original work is properly cited.

\begin{abstract}
Exosomes are a type of extracellular vehicle, formed by budding cell membranes, containing proteins, DNA, and RNA. Concentrated cargoes from parent cells are enveloped in exosomes, which are cell specific and may have functions in the recipient cell, reflecting a novel physiological and pathological mechanism in disease development. As a transmitter, exosomes shuttle to different cells or tissues and mediate communications among these organelles. To date, several studies have demonstrated that exosomes play crucial roles in disease pathogenesis and development, such as breast and prostate cancer. However, studies investigating connections between exosomes and thyroid disease are limited. In this review, recent research advances on exosomes in thyroid cancer and Graves' disease are reviewed. These studies suggest that exosomes are involved in thyroid disease and appear as impressive potentials in thyroid therapeutic areas.
\end{abstract}

\section{Exosomes}

Exosomes are extracellular vehicles (EVs) with approximate sizes of $40-100 \mathrm{~nm}$ with a density of $1.13-1.19 \mathrm{~g} / \mathrm{ml}$ in sucrose density gradients; formed by a process of endosome membrane intracellular invagination [1], exosomes are released from patient cell, transmitting into circulation and specific sites (Figure 1). In 1987, these membrane vesicles were initially observed in rat reticulocytes [2] and termed "exosomes" [3]. Exosome cargoes are varied, including lipids [4], DNA [5], proteins [6], growth factors [7], and several types of RNAs [8-10], such as microRNA (miRNA), long noncoding RNA (lncRNA), circular RNA (circRNA), and mRNA. The mechanism of those cytosolic components selectively transferred into exosomes is not revealed clearly. Recently, the endosomal proteins such as Hsc70 and Hsp90 could potentially play a role in exosomal protein sorting [9]. Furthermore, the endosomal sorting complex responsible for transport (ESCRT)-II and specific sequence motifs shared by RNAs in exosomes may be associated with RNA sorting $[10,11]$. It had been exhibited that exosomes virtually exist in all bodily fluids, including serum, plasma, and urine [12-14].

1.1. The Function of Exosomes. Diverse exosomal functions have been described, chiefly including serving as transmitters shuttling between different cells and mediating intracellular communications, such as cellular differentiation, promoting angiogenesis, and modulating immune responses [15-20]. These functions can be divided into two types: disease development and disease reversal.

Exosome cargoes, when targeted to recipient cells, can behave as disease contributors. A crucial role of exosomes in HIV infection revealed recently [21]. Chiozzini et al. observed that when dendritic cells (DC) mediated the trans-dissemination of exosomes from $\mathrm{HIV}$-1-infected cells to resting $\mathrm{CD}^{+}$ $\mathrm{T}$ lymphocytes, the process induced efficient trans- 


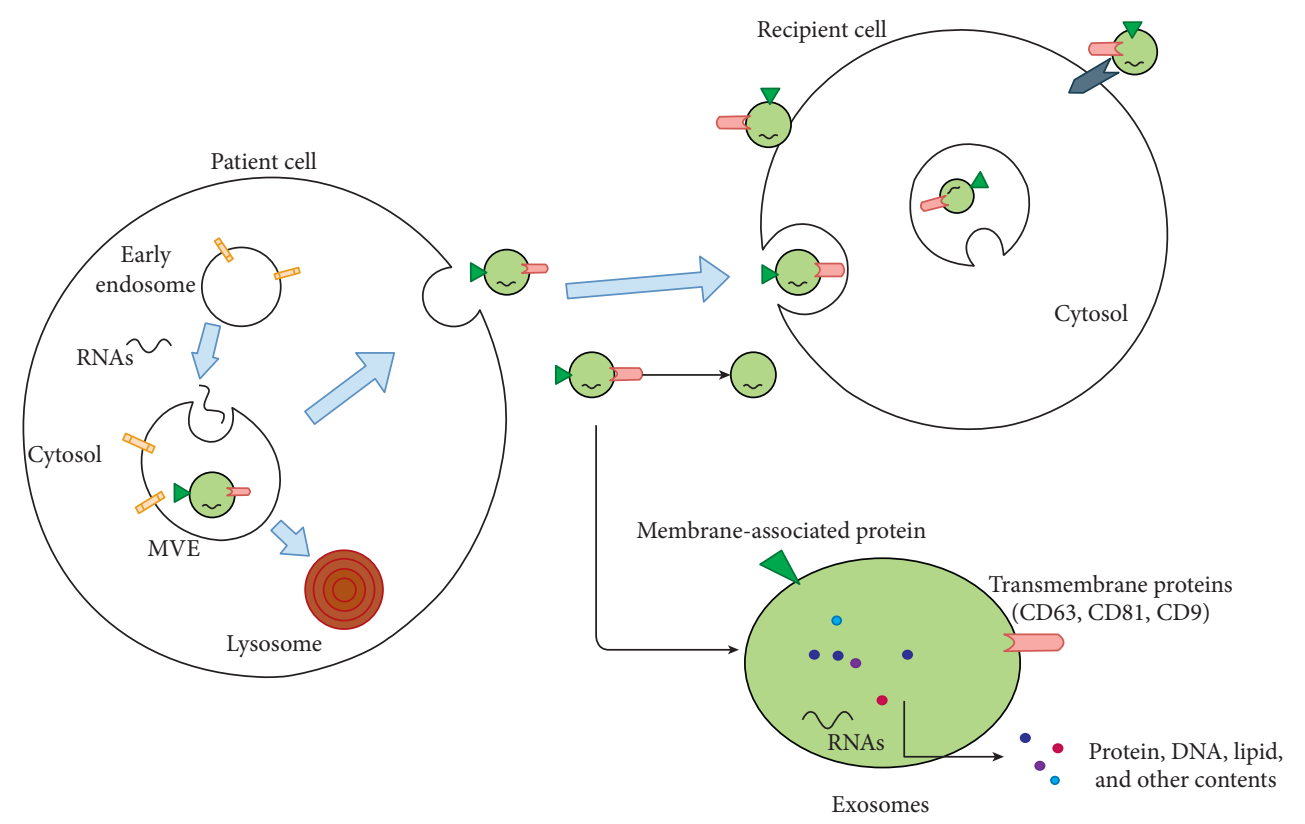

FIGURE 1: The formation and transmitting of exosomes. Exosomes are formed as the vehicles by budding into multivesicular endosomes (MVEs) and then released by the fusion of MVEs with the plasma membrane. Other MVEs proceed into lysosome pathway for degradation. Exosomes released from patient cells transmitting to recipient cells affect recipient cells by direct fusion with membrane of recipient cells, endocytic uptake, and ligand-mediated interaction. CD63, CD81, and CD9 are common surface biomarkers of exosomes. RNAs, protein, DNA, and other contents encased into exosomes can be transmitted into target cells.

dissemination and HIV-1 expression in target cells [22]. Yi et al. observed that miR-146a in exosomes suppressed type I interferon responses and facilitated EV71 infection [23]. Ye et al. uncovered that exosomes derived from celecoxib on nasopharyngeal carcinoma TW03 cells generated T-cell dysfunction, by inhibiting T-cell proliferation and Th1 and Th17 differentiation and promoting Treg induction in nasopharyngeal carcinoma cells [24]. These results suggest that exosomes secreted from parental cells have impact on the function of immune cells, especially T cells.

Angiogenesis plays an important role in oncogenesis and metastasis. Kosaka et al. showed that upregulation of miRNA$351 \mathrm{~b}$ and miRNA-210 in exosomes released by cancer cells, resulted in enhanced tumor angiogenesis [25]. Hsu et al. observed that miRNA-23a in exosomes derived from lung cancer cells increased tumor angiogenesis under hypoxic and normal conditions [26]. These observations show that miRNAs in exosomes secreted from cancer cells enhance angiogenesis, promoting tumor development in cancer.

Proteins harboured in exosomes appeared to facilitate disease procedures as well. Blomme et al. showed that myoferlin was overexpressed in exosomes derived from different breast and pancreatic cancer cell lines, promoting human umbilical vein endothelial cell (HUVEC) migration and invasion. In contrast, myoferlin-depleted exosomes exhibited decreased abilities in inducing HUVEC proliferation and migration [27]. Additionally, proteins attached to exosome cell surfaces contribute to exosomal functions. Christianson et al. showed that heparan sulfate proteoglycans (HSPGs) function as internalizing receptors of exosomes, inhibiting exosomemediated migration of cancer cells through proteoglycans in proteoglycan-deficient cells [28]. These data demonstrate that proteins in exosomes derived from cancer cells, directly or indirectly, affect tumor migration.

Similarly, exosomes function in disease resistance. Exosome cargoes can act as mitigating factors in several molecular scenarios. Zhao et al. found that exosomes containing active signal transducers and activators of transcription 3 (STAT3) produced by adipose-derived stem cells could be transferred into macrophages, inducing anti-inflammatory M2 phenotypes through transactivation of arginase-1 [29]. Song et al. revealed that miRNA-146, a well-known anti-inflammatory factor, packaged in exosomes derived from mesenchymal stem cells, targeted macrophages, resulting in anti-inflammatory M2 phenotype formation [30]. It appears that anti-inflammatory M2 phenotypes generated by exosomal mechanisms are efficient in assisting inflammatory disorder reversal. Exosomes play a role in cardiovascular system as well. Our present study investigated that exosomes derived from melatonin-treated vascular smooth muscle cells could mitigate vascular calcification and aging through an exosomal miR-204/miR-211 cluste [31]. Cheng et al. proposed a mechanism whereby miRNAs enveloped in impaired myocardium-derived exosomes can be selectively transferred into bone marrow cells to mobilise progenitor cell migration to lesions, promoting heart tissue repair [30]. These discoveries indicate a key property of exosomes in damage resistance and healing acceleration at damaged sites. In conclusion, exosomes contribute to an enhanced interpretation of cell physiology and pathology.

\section{Thyroid Diseases}

Thyroid diseases can be broadly categorized as hypothyroidism, hyperthyroidism, goiter, and cancers. Both 
hyperthyroidism and hypothyroidism are thyroid dysfunctions, presenting symptomatic and readily diagnosed by laboratory data of thyroid hormones, which can be treated under further investigation of clear and definite causes. The most clinically important thyroid diseases are thyroid cancer (TC) and thyroid goiter. As the most prevalent malignancy of the endocrine system, TC represents approximately $1.5 \%$ to $2.1 \%$ of all cancers diagnosed annually worldwide, being divided into differentiated carcinoma, which includes papillary thyroid cancer (PTC), follicular and Hurthle cell cancer [33], and undifferentiated carcinoma, which consists of medullary thyroid cancer (MTC) and anaplastic thyroid cancer (ATC) [34]. PTC accounts for $80-85 \%$ of all TCs. According to clinical statistics, young females are the main targets for PTC [35]. Even though comparatively satisfactory PTC prognosticated in the clinic, a small portion of PTC turns aggressive, meaning the tumor is capable of developing into distant metastases, thereby increasing the death rate [36]. With the highest mortality rate, ATC exists as the most aggressive tumor of these four types [37]. Up to now, the main problem with TC treatment was early and overdiagnosis, as well as neoplasm staging, all of which could be resolved to a large extent by exosome biology.

Thyroid goiter comprises nontoxic or toxic conditions, bringing inconvenience to patients and lowering their quality of life. Reports have recommended that globally goiter is mainly caused by iodine deficiency, while several other factors give rise to thyroid goiter as well, such as multinodular thyroid disease, chronic autoimmune thyroiditis, and Graves' disease (GD) [38, 39]. GD deserves further research as it seriously impacts the quality of life.

\section{Exosomes in TCs}

Even as a common endocrine malignancy, the specific pathogenesis/tumorigenesis of TC remains unclear. Up to now, more than a few TC mechanisms have been elucidated, involving several signalling pathways, such as the mitogenactivated protein kinases (MAPK) pathway, and the phosphatidylinositol-3-kinase and protein kinase B (PI3K-AKT) pathway, and molecular rearrangements such as mutation, gene copy-number gain, and aberrant gene methylation [40]. It had been proposed that cargoes harboured in exosomes are connected to signalling pathways. Additionally, explorative connections between exosomes biology and cancers, such as breast, prostate, ovarian, and lung cancer, are ongoing. Similarly, the relationship between exosomes and thyroid disease has lately emerged; therefore, do exosomes play biological roles in thyroid-related diseases, and furthermore could exosomes perform potential functions in thyroid disease therapy?

\subsection{Variation in Expression of Exosomal Cargoes in TC.} Exosomes function in cell-to-cell communications, including normal cells to cancer cells. Several studies have revealed that exosome cargos are alterable as well as cargo quantities depend on the disease process. It appears that cargo up- or downregulation makes crucial differences to biological outcomes.
Lee et al. proposed that PTC cell line cell-derived exosomes contained overexpressed miRNA-146b and miRNA222 molecules, when compared with normal thyroid cells. Furthermore, reductions of meaningful fold of these two miRNAs levels after total thyroidectomy were detected in the plasma, correspondingly [41]. Roman et al. showed that miRNA-31-5p, miRNA-146a-5p, miRNA-21-5p, miRNA221-3p, and miRNA-181a-5p were statistically overexpressed in PTC-derived exosomes. Precisely, overrepresented miRNA-31-5p was found when compared with benign tumors while miRNA-146a-5p, miRNA-21-5p, miRNA-221-3p, and miRNA-181a-5p were increased corresponding to other TC types [42]. Similarly, another study found that miRNA-21 and miRNA-181a in PTC-derived exosomes were differentially expressed. Yang et al. revealed that 22 circular RNAs (circRNAs), in serum exosomes derived from PTC patients, were differentially expressed, when processed by highthroughput sequencing. Compared with the control group, three circRNAs including hsacirc_007293, hsacirc_031752, and hsacirc_020135 were upregulated, and 19 were downregulated [43]. Wang et al. demonstrated that miR346 , miR-10a-5p, and miR-34a-5p were upregulated in PTC-derived exosomes [44]. Differential expression of miRNAs in exosomes derived from PTC patients may indicate different functions in PTC pathogenesis. Variations in mechanisms and pathways have been investigated. According to DIANA-Tarbase analysis, miR-346 may be associated with several pathways, including the TC p53 signalling pathway, mammalian target of rapamycin (mTOR) signalling pathway, and the MAPK signalling pathway. MiR-10a-5p was found to interact with cell cycle, p53, and MAPK signalling pathways in TC pathogenesis [44]. Majority information was focused on the variation of miRNA, whereas few detections concerned with rest of substances encapsulated within exosomes had been discovered. Edo et al. proposed that exosomes expressing the thyrotropin receptor (TSHR) may be secreted from normal and cancerous thyroid cells [45]. Luo et al. found 697 differentially expressed proteins in serum-purified exosomes (SPEs) of PTC patients with lymph node metastasis (LNM), when compared with SPEs from PTC patients without LNM [46]. While mechanisms have not been fully revealed, these observations provide clues that exosomal cargoes are intentionally delivered into selected cells. Further studies are required to investigate exosomal cargoes and their significance in pathophysiology.

3.2. The Role of Exosomal Cargoes in TC Development. As the differential expression of exosomal cargoes has been proposed, further studies revealed their significance and mechanism. It has been suggested that exosomes derived from original cells, containing certain molecules, can transport these cargoes to target cells near and far, thereby causing tumor development. Several exosomal mechanisms have been proposed for the development of TC (Figure 2).

Luo et al. revealed that SRC, TLN1, ITGB2, and CAPNS1 proteins were overexpressed in PTC-derived exosomes, accelerating tumor metastasis. Furthermore, integrin-associated 


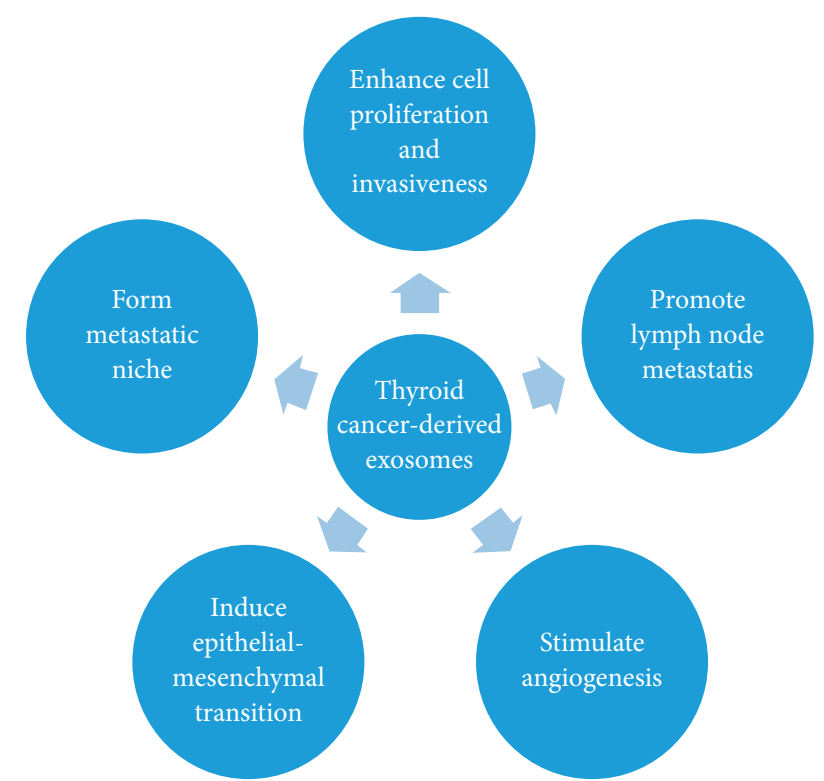

Figure 2: The role of TC-derived exosomes in cancer development. Exosomes derived from thyroid cancer cells promote the development of TC by direct and indirect ways. Direct approaches include enhance cell proliferation and invasiveness and promote lymph node metastasis. Indirect approach includes form metastatic niche, stimulate angiogenesis, and induce epithelial-mesenchymal transition to support tumor progress.

proteins were upregulated in SPE from PTC with LNM, when compared with patients without LNM [46]. Our recent study suggested that exosomes isolated from hypoxic PTC, BCPAP, and KTC-1 cells contained increased miRNA-21-5p levels, which contributed to HUVEC angiogenesis. Angiogenesis was attenuated after the knockdown of miRNA-21-5p in exosomes. Our study revealed a new mechanism of hypoxic PTC angiogenesis, via the exosomal miRNA-21-5p/TGFBI and miRNA-21-5p/COL4A1 pathways, suggesting an original perspective for PTC therapy [47]. Ye et al. demonstrated that exosomal miRNA-432-5p in PTC serum exosomes was significantly overexpressed and associated with lymph node metastasis; the capacity for PTC cell migration and invasion was enhanced by exosomal miRNA-432-5p [48]. These results suggest that protein and miRNA levels in exosomes are associated with TC stage and LNM, which hit the disease procedure and prognosis.

Epithelial-mesenchymal transitions (EMT) occur under the influence of exosomal cargoes as well. The EMT involves epithelial cells that undergo a transition to a mesenchymal phenotype, characterised by the loss of E-cadherin, which is activated by factors such as SNAIL, SLUG, ZEBs and TWIST [36]. Cells gain the ability to transfer and invade other cells, promoting the metastasis of primary tumours to develop into secondary tumours [36]. Studies have suggested that in thyroid tumours, the induction of EMT was associated with the overexpression of vimentin, related to PTC. Additionally, the invasion and lymph node metastasis of PTC occurs through EMT [49]. The loss of E-cadherin and membranous $\beta$-catenin, and the activation of SLUG and TWIST mechanisms are linked to undifferentiated carcinomas, especially ATCs $[50,51]$.

A study found that thyroid cancer stem-like cell (CSC) derived exosomes, transferred lncRNA, specifically lincROR, to trigger EMT, inducing local tumor microenvironments and distant metastatic niches [36]. In contrast, exosomal miRNA-145 suppressed the growth and transfer of TC cells; Compared with the healthy group, the expression of exosomal miR-145 was significantly decreased in TCs. Additionally, the upregulation of miR-145 in TC cells triggered decreased cell proliferation, migration, invasion, vascular endothelial growth factor (VEGF) secretion and E-cadherin expression, by targeting the PI3K/Akt pathway [52]. In conclusion, the function of exosomal contents can be seen in Table 1.

3.3. Exosomes as Fluid Biopsy in TC Diagnosis and Therapy. In different pathological states and stages, abundant exosomes encapsulating different substances exist in the circulation. Most of these variations are corresponding with the pathological stages, suggesting analysis of the differential expression of these cargoes may provide novel outlooks on TC diagnosis and therapy.

3.3.1. Exosomal-Mediated Therapies. Because endosomes appear to differentially promote disease development and disease resistance, their potential exploitation as disease treatments has aroused the interest of many researchers. Further exploratory studies have focused on therapeutic areas, where exosomes act as delivery tools to transport drugs to targeted areas. Several studies concerning exosome therapeutic effects in cancer diseases $[53,54]$, inflammatory diseases [53], and even in psychological illness [56] emerged.

Hoshino et al. found that tumor-secreted exosomes, which was uptaken by organ-specific cells to prepare the premetastatic niche, played an important role in tumor metastasis. Also, specific exosomal integrin expression pattern was related to different tumor metastases [55]. Richards et al. revealed treatment of cancer-associated fibroblasts with an inhibitor of exosome biogenesis GW4869 attenuated chemoresistance in cocultured cancer cell in vitro and inhibited cancer growth in vivo [56]. These observations imply that blocking exosome biogenesis and secretion could be a feasible approach for disease therapy.

In order to deliver targeted drug, Qi et al. developed a exosome-based superparamagnetic nanoparticle cluster which can serves as drug-transmitting vehicles. The results showed that reticulocyte-derived doxorubicin-loaded exosomes could facilitate cancer targeting in an external magnetic field and suppress tumor growth [57]. Additionally, Xiao et al. suggested that tumor exosome-loaded dendritic cells could activate and recruit effector T cells, and prolong the survival time of pancreatic cancer patients, when combined with cytotoxic drug treatments [58]. These results suggest that exogenous molecules can be carried by exosomes and transferred to targeted areas. 
TABLE 1: Role of exosomal contents in TC development.

\begin{tabular}{|c|c|c|c|c|c|}
\hline Exosomal origin & Author & Contents & Function & Level & Reference \\
\hline Serum & Luo et al. & SRC & $\begin{array}{l}\text { Regulate tumor metastasis via activating MAPK/ERK and } \\
\text { focal adhesion kinase (FAK) pathways }\end{array}$ & Overexpress & {$[46]$} \\
\hline Hypoxic PTC cells & Wu et al. & $\begin{array}{l}\operatorname{miRNA}-21- \\
5 \mathrm{p}\end{array}$ & $\begin{array}{l}\text { Via miRNA-21-5p/TGFBI and miRNA-21-5p/COL4A1 } \\
\text { pathway to promote angiogenesis of HUVECs }\end{array}$ & Overexpress & {$[47]$} \\
\hline PTC serum & Ye et al. & $\begin{array}{c}\text { miRNA- } \\
432-5 \mathrm{p}\end{array}$ & Accelerating the lymph node metastasis & Overexpress & {$[48]$} \\
\hline TC-derived cell lines & Hardy & SNAIL & $\begin{array}{l}\text { Repressing CDH1 transcription and promoting the } \\
\text { development of PTC }\end{array}$ & Overexpress & {$[49]$} \\
\hline CSCs & Hardin & Linc-ROR & $\begin{array}{l}\text { Trigger EMT and induce the local tumor microenvironment } \\
\text { and the distant metastatic niche }\end{array}$ & Overexpress & {$[36]$} \\
\hline $\begin{array}{l}\text { TPC- } 1 \text { cell lines and } \\
8505 \text { cells }\end{array}$ & Boufraqech & miR-145 & $\begin{array}{c}\text { Via inhibiting the PI3K/Akt pathway to suppress the growth } \\
\text { and transfer of TC }\end{array}$ & Downexpress & {$[52]$} \\
\hline
\end{tabular}

3.3.2. Fluid Biopsy in TC. Fluid biopsy is a novel concept which consists of circulating tumor DNA (ctDNA), circulating tumor cells (CTCs), and exosomes [59]. There are several drawbacks to traditional tissue biopsy, such as its invasiveness, difficulties in repeat sampling, and implantation metastasis occurrence. As a noninvasive and safe approach, fluid biopsy brings a new perspective for tumor screening and diagnosis. Fluid biopsy provides a genetic snapshot of the tumor landscape, including both primary and metastatic loci [60].

TC is the most common cancer in American adults, aged between 16 and 33 years [61]. With a low fatality rate, but high and increasing incidence, the overdiagnosis of TC frequently occurs $[38,62,63]$. Routine diagnosis of thyroid nodules usually relies on ultrasound-mediated fine-needle aspirate biopsy (FNAB), which is invasive and often inaccurate Two challenges exist in TC diagnosis: (1) the identification of malignant thyroid nodules and benign nodules. Thyroid nodules are one of the most common symptoms in thyroid disease; approximately $15 \%$ of thyroid nodules are malignant [64]. Traditional methods guided by ultrasound [65] give rise to misdiagnoses, causing frequent overdiagnoses and overtreatments [66]. (2) Confirmation of the TC stage involved with LNM. Therapy methods for TC depends on tumor metastasis progression. Up to $50 \%$ neck-lymph node involved in TC patients and half of those nodes may be misjudged by neck-ultrasound. Isolation of genetic materials from human fluid is a minimally invasive method, leaving liquid biopsy a stage in the clinical approach to TC diagnosis.

The protein profiling is challenging for its small size, low abundance, and heterogeneity [67]. Still few researches found protein expressed significantly different among TC patients could be serve as biomarkers applied to improve diagnostic accuracy noninvasively. Fucosylation is one of the most crucial types of glycosylation involved in carcinogenesis. Comparing glycoproteomic difference of human body between healthy controls and patients with PTC, studies investigated that 44 fucosylated and 59 nonfucosylated desialo-N-glycopeptides were differentially expressed in patients, the ratio of fucosylated to fucosylated structures for each N-glycopeptide (F/NF) were correspondingly upregulated or downregulated in PTC, suggesting the F/NF ratio is a novel diagnostic marker of PTC
[68]. Lin et al. publicised that 15 proteins were well distinguished between patients with LNM and patients without LNM. Additionally, the attenuation of tumor growth when knocking down ISG15 with shRNA implied that ISG15 probably serves as a prognosis indicator in fluid biopsy of thyroid papillary microcarcinoma patients with LNM [69]. Pazaitou-Panayiotou et al. found that ratios of insulin-like growth factor (IGF-1) to adiponectin and IGF-1 to (adiponectin $\times$ IGF-binding protein 3 ) were positively associated with tumor size even though the level of these three proteins had no obvious difference among several histologic types of TC [70]. These results definitely present the probably use of proteins in fluid biopsy in TC.

Studies focus on miRNAs in TC body fluids hint that miRNAs are the most potential circulating biomarkers for cancer diagnosis. The diagnosis of PTC persistence or recurrence, which cause significant inconvenience and trouble in patients, might benefit from fluid biopsy. Thyroglobulin is considered to be a main biomarker for detecting persistent/ recurrent thyroid cancer. MiRNAs hold remarkable promise as serum biomarkers for monitoring TC patients when thyroglobulin assay results are uninformative. Rosignolo et al. discovered that circulating levels of miR-146a-5p and miR-221-3p were downregulated after tumor resection and investigated these molecules in serum as potential biomarkers for the early noninvasive detection of persistent PTC [71]. Another study demonstrated that miR-222 and miR-146b were associated with PTC recurrence; miR-222 and miR-146b circulatory levels corresponded to the presence of PTC [41]. These data suggest that different miRNAs could be markers of PTC. The distinct expression of miR-95 and miR-190 in PTC patient serum shows that miR-190 is upregulated, whereas miR-95 is downregulated, suggesting that these molecules act as complementary biomarkers for the differential diagnosis of thyroid nodules [72]. Li et al. suggested that levels of plasma miR-25-3p and miR-451a were significantly decreased after tumor excision from PTC patients. ROC analyses showed that plasma miR-25-3p and miR-451a levels had sensitivities of $92.8 \%$ and $88.9 \%$, and specificities of $68.8 \%$ and $66.7 \%$, respectively, in distinguishing PTC from benign nodules [73]. Another study revealed that the upregulation of both miR-146b-5p and miR-146b-3p characterised PTC-classical-type (CT) and 
PTC-tall-cell variant (TCV), but not PTC-follicular-variant (FV), whereas miR-21-5p was significantly overexpressed in PTC-TCV only. Also, miR-204-5p was downregulated in all histological subtypes of PTC-FV, except PTC-encapsulated follicular variant (EFV) [74]. These results demonstrate novel perspectives for the molecular classification of PTC, showing that different miRNA expression profiles are associated with different PTC histological types. Clearly, miRNA levels in the bloodstream of TC patients are fluctuant, indicating enormous potential for TC diagnostics and therapeutics.

3.3.3. Exosome-Based Fluid Biopsy in TC. Exosomes exist in almost all fluids of the human body, such as plasma, serum, and urine. Due to their predominant status in blood flow, exosomes show tremendous advantages when compared with CTCs and ctDNA [75]. They exhibit their effects not only in pathogenic processes but also in therapeutic processes where a huge potential has been revealed. It has been reported that exosomes are now important fluid biopsy tools in several diseases, such as infections [21] and cancers like ovarian [76], prostate [77], gastric [78], breast [79], and brain tumours [80]. Although few research studies have been reported on exosomes released by thyroid cancer, experimental evidence exists to support the involvement of exosome contents especially miRNAs in thyroid cancers, showing the hypothesis that exosomes serve as fluid biopsy tools, for diagnosing and identifying different TC types.

Wang et al. investigated the upregulation of miR-346, miR-10a-5p, and miR-34a-5p in PTC plasma exosomes, establishing a three-miRNA plasma panel with huge potential clinical benefits in discriminating PTC from healthy controls, or nodular goiter [44]. Boufraqech $\mathrm{M}$ et al. reported that screening decreased exosomal miR-145 level of PTC and ATC could be regarded as an effective test in patients with inconclusive FNABs [52]. Comparing miRNA profiles of patients with recurrent PTC and those without recurrence, Lee et al. found that miRNA-146b and miRNA-222 in PTC-derived exosomes were overexpressed for about 10fold, suggesting those miRNAs may be developed into alternative biomarkers for PTC recurrence [41, 81]. Exosomal miRNA profiling has made contributions to distinguishing PTC from follicular thyroid carcinoma (FTC). Studies have shown that miR-21-5p and miR-221-3p were upregulated in FTC-derived exosomes, while miR-181a was upregulated in PTC-derived exosomes. The ratio of miR-21-5p/miR-181a showed high sensitivity in identifying FPC and PTC. The combination of miR-21-5p/miR-181a and miR-221-3p/miR181a ratios improves the specificity of identification. These observations indicate that the expression of miR--21-5p, miR-221-3p, and miR-181a has serious clinical applications for the diagnosis of differentiated TC [42] (Table 2).

More than a few cohort studies are necessitated to investigate exosomes as circulating biomarkers for TC diagnosis and treatment. Correlational studies are concentrated on exosomal miRNAs released by TCs. Up to now, investigations around other contents of exosomes may provide further evidence and strengthen the perspective in the future. Beyond all doubt, fluid biopsy will present huge capacity and superiority to play a critical role in therapeutic and diagnostic area in cancer diseases, bringing extraordinary benefits to clinical medicine to a large extent.

\section{Exosomes in GD}

GD is the most frequent thyroid goiter-related disease, accounting for $50-80 \%$ of hyperthyroidism [82]. Populations with GD mainly consist of females, aged between 30 and 50 years. GD comprises an enlarged and overactive thyroid gland and increased heart rate and proptosis [83]. Typical GD manifestation results from thyroid hormone overproduction, which is related to sensitised thyrotropinreceptor antibodies. GD is a chronic disease, similar to diabetes and heart disease, where long-term medical treatment is required, which is lack of therapies against primary pathogenic mechanisms [82]. GD diagnosis is relatively straightforward; nevertheless, therapeutic remedies are more perplexing. Despite great progress and development in current understanding of the cellular and molecular basis of autoimmunity, the current treatments for GD have been at best invariable over the last 50 years [84]. Typical treatments include antithyroid medications, radioactive iodine ablation, and surgery [85]. GD deserves further research as it seriously impacts the quality of life.

Connections between exosomes and GD pathogenesis have been investigated, since it was first discovered that exosomes are vehicles shuttling back and forth in various cells. Hiratsuka et al. collected exosomes from intractable GD patients, which could stimulate mRNA expression for IL-1, TNF-a, and IL-6 in peripheral blood mononuclear cells compared with GD patients in remission or healthy controls, thereby activating immune reactions. This finding suggested that exosomes may play roles in GD pathogenesis [86].

In healthy individuals, the proliferation of thyroid cells and synthesis and secretion of thyroid hormones are regulated by a combination of TSH and its receptors. However, autoantibodies targeting the TSHR, mimic the actions of TSH, causing thyroid hyperfunction in GD patients. Edo et al. provided evidence that TSHR is detected in exosomes, secreted from normal and cancerous thyroid cell lines, and may exert decoy effects by binding to autoantibodies, thus ameliorating autoantibody-mediated activation in GD patients [45]. Interestingly, another study by Rossi et al. revealed that dichlorodiphenyltrichloroethane (DDT)-induced formation and shift of exosomes containing TSHRs dislodged from thyroid cells could directly stimulate lymphocytes produced by TSH receptor-stimulating autoantibodies, leading to the development of GD [87]. These results appear to be contradictory; therefore, they require further investigation on the function of TSHRs loaded in exosomes. Probably, these observations are caused by diverse circumstances. For example, the effect of TSHR is likely to be completely conflicting under physiological and pathological conditions.

\section{Summary and Perspectives}

As a crucial component of the extracellular vascular matrix, exosomes exist almost everywhere in circulation. Since their 
TABle 2: Potential biomarkers of exosomal miRNAs in different types of TC.

\begin{tabular}{lccc}
\hline Origin & Potential biomarker & Expression level & Reference \\
\hline PTC plasma exosomes & miR-346, miR10a-5p, and miR34a-5p & Up & {$[44]$} \\
PTC- and ATC-derived exosomes & miR-145 & Down & Up \\
PTC-derived exosomes & miRNA-146b and miRNA-222 & Up & {$[52]$} \\
FTC-derived exosomes & miR-21-5p and miR-221-3p & $\mathrm{Up}$ & {$[41,81]$} \\
PTC-derived exosomes & miR-181a & {$[42]$} & {$[42]$} \\
\hline
\end{tabular}

discovery, this small particle has been enigmatic, attracting researchers to get to the bottom of their mysteries. From our unequivocal knowledge, these molecules are secreted by all kinds of cells and then released into circulation as vehicles, transferring messages/cargoes from original cells to the target site. The exosomal sorting mechanisms as well as the fluctuant level of components appear to numerous scholars looking for their eternal secrets. Dramatically, exosomes emerged with dual characters. On the pernicious side, along with the delivery of exosomes, detrimental productions, such as inflammatory factors from pathogens and metabolites from necrotic tissues, interacted with target cells. Acting as a quiet accomplice, exosomes trigger further injuries in human body. Majority of researches focused on exosomal miRNAs and protein levels which turned out significantly variation during pathogenesis, opening a window for deciphering diseases mechanisms. Furthermore, blocking exosomal secretion from patient cells and transferring pathways of these cargos offer a breakthrough for disease therapy.

Alternatively, exosomes display antipathogenic activities, accompanied by releasing factors alleviating tumour development. Nevertheless, the majority of these pathways have not been unearthed, which demands more attention. Additionally, increasing discovered evidence concerning exosomes to be used as delivery vehicles for disease treatment indicates a huge potential for therapeutic areas. Undoubtedly, the enormous prospective of exosomes deserves additional investigation.

Thyroid-related diseases primarily consist of TC, thyroid goiter, and thyroid dysfunction, which include hypothyroidism and hyperthyroidism. Thyroid dysfunction can be dexterously diagnosed by clinical features and laboratory data, and further therapies are regularly intervened by medicine controlling. On the contrary, challenges exist in the stage and diagnosis of thyroid goiter and carcinoma. In addition, pathogenic and aggravating mechanisms of GD are still unclear. The function of exosomes brings light to these complexities. Recently, data showed that cargoes encapsulated in exosomes appeared to vary in patients with thyroid disease. Accordingly, several studies about the pathway of changing mechanisms revealed partial causes, thereby inducing further imagination about the application of exosomes. Most of those variation is significantly related to disease progression and prognosis, giving clues for diagnosis and treatment of thyroid diseases. Altered level of exosomal cargos correspond to distinctive pathological stage. Combination of several biomarkers makes TC diagnosis and treatment more conclusiveness.

Fluid biopsy is a novel technique which exhibits momentous potential for thyroid disease diagnosis and therapy.
Even though miscellaneous, other irrelevant fluid components existing in circulation may cause instability to the detection results. Several liquid biopsy companion diagnostic tests approved by the regulatory agencies for the selection of patients in clinical practice light on an innovative way for fluid biopsy. In recent decades, tremendous advances and progress have been made in probing exosomes as regulators of thyroid disease. Incontrovertibly, the combination of exosomes and fluid biopsy creates a perspective for TC therapeutic field. However, up-to-date results are notwithstanding at the tip of the research iceberg. With advances in technology and more controlled, largescale, and cohort-driven studies, unravelling the promise of exosomes to play its therapeutic potential for thyroid disease is just around the corner.

\section{Abbreviations}

EVs: $\quad$ Extracellular vehicles

miRNA: MicroRNA

lncRNA: Long noncoding RNA

circRNA: Circular RNA

ESCRT: Endosomal sorting complex responsible for transport

MVEs: $\quad$ Multivesicular endosomes

DC: Dendritic cells

HUVEC: Human umbilical vein endothelial cell

HSPGs: Heparan sulfate proteoglycans

STAT3: Signal transducers and activators of transcription 3

TC: $\quad$ Thyroid cancer

PTC: $\quad$ Papillary thyroid cancer

MTC: $\quad$ Medullary thyroid cancer

ATC: Anaplastic thyroid cancer

GD: $\quad$ Grave disease

MAPK: Mitogen-activated protein kinases

PI3K-AKT: Phosphatidylinositol-3-kinase and protein kinase $\mathrm{B}$

circRNAs: Circular RNAs

mTOR: Mammalian target of rapamycin

TSHR: Thyrotropin receptor

SPEs: $\quad$ Serum-purified exosomes

LNM: $\quad$ Lymph node metastasis

EMT: $\quad$ Epithelial-mesenchymal transitions

CSC: $\quad$ Cancer stem-like cell

VEGF: Vascular endothelial growth factor

FAK: $\quad$ Focal adhesion kinase

ctDNA: Circulating tumor DNA

CTCs: Circulating tumor cells

FTC: $\quad$ Follicular thyroid carcinoma 
IGF-1: Insulin-like growth factor

FNAB: $\quad$ Fine-needle aspirate biopsy.

\section{Conflicts of Interest}

The authors declare that they have no conflicts of interest.

\section{Acknowledgments}

This work was funded by the National Natural Science Foundation of China (grant nos. 81770881 and 81870623) and the Fundamental Research Funds for the Central Universities of Central South University (grant no. 2018zzts048).

\section{References}

[1] X. Zhang, X. Yuan, H. Shi, L. Wu, H. Qian, and W. Xu, "Exosomes in cancer: small particle, big player," Journal of Hematology \& Oncology, vol. 8, no. 1, p. 83, 2015.

[2] C. Harding and P. Stahl, "Transferrin recycling in reticulocytes: $\mathrm{pH}$ and iron are important determinants of ligand binding and processing," Biochemical and Biophysical Research Communications, vol. 113, no. 2, pp. 650-658, 1983.

[3] R. M. Johnstone, M. Adam, J. R. Hammond, L. Orr, and C. Turbide, "Vesicle formation during reticulocyte maturation. Association of plasma membrane activities with released vesicles (exosomes)," The Journal of Biological Chemistry, vol. 262, no. 262, pp. 9412-9420, 1987.

[4] T. Skotland, K. Ekroos, D. Kauhanen et al., "Molecular lipid species in urinary exosomes as potential prostate cancer biomarkers," European Journal of Cancer, vol. 70, pp. 122132, 2017.

[5] A. Takahashi, R. Okada, K. Nagao et al., "Exosomes maintain cellular homeostasis by excreting harmful DNA from cells," Nature Communications, vol. 8, p. 15287, 2017.

[6] M. Pietrowska, S. Funk, M. Gawin et al., "Isolation of exosomes for the purpose of protein cargo analysis with the use of mass spectrometry," Methods in Molecular Biology, vol. 1654, pp. 291-307, 2017.

[7] S. Zhang, S. J. Chuah, R. C. Lai, J. H. P. Hui, S. K. Lim, and W. S. Toh, "MSC exosomes mediate cartilage repair by enhancing proliferation, attenuating apoptosis and modulating immune reactivity,” Biomaterials, vol. 156, pp. 16-27, 2018.

[8] D. Enderle, A. Spiel, C. M. Coticchia et al., "Characterization of RNA from exosomes and other extracellular vesicles isolated by a novel spin column-based method," PLoS One, vol. 10, no. 8, Article ID e0136133, 2015.

[9] S. I. Buschow, E. N. M. Nolte-'t Hoen, G. Van Niel et al., "MHC II in dendritic cells is targeted to lysosomes or T cellinduced exosomes via distinct multivesicular body pathways," Traffic, vol. 10, no. 10, pp. 1528-1542, 2009.

[10] A. O. Wauben, V. A. Kuznetsov, and I. V. Kurochkin, "Identification of nucleotide patterns enriched in secreted RNAs as putative cis-acting elements targeting them to exosome nano-vesicles," BMC Genomics, vol. 12, no. Suppl 3, p. S18, 2011.

[11] U. Irion and D. St Johnston, "Bicoid RNA localization requires specific binding of an endosomal sorting complex," Nature, vol. 445, no. 7127, pp. 554-558, 2007.

[12] Y.-T. Tang, Y.-Y. Huang, L. Zheng et al., "Comparison of isolation methods of exosomes and exosomal RNA from cell culture medium and serum," International Journal of Molecular Medicine, vol. 40, no. 3, pp. 834-844, 2017.

[13] H. Wang, C. G. Adda, M. Liem et al., "Comparative proteomics evaluation of plasma exosome isolation techniques and assessment of the stability of exosomes in normal human blood plasma," Proteomics, vol. 13, no. 22, pp. 3354-3364, 2013.

[14] M. Li, E. Zeringer, T. Barta, J. Schageman, A. Cheng, and A. V. Vlassov, "Analysis of the RNA content of the exosomes derived from blood serum and urine and its potential as biomarkers," Philosophical Transactions of the Royal Society B: Biological Sciences, vol. 369, no. 1652, Article ID 20130502, 2014.

[15] J. Zhang, S. Li, L. Li et al., "Exosome and exosomal microRNA: trafficking, sorting, and function," Genomics, Proteomics \& Bioinformatics, vol. 13, no. 1, pp. 17-24, 2015.

[16] R. Chowdhury, J. P. Webber, M. Gurney, M. D. Mason, Z. Tabi, and A. Clayton, "Cancer exosomes trigger mesenchymal stem cell differentiation into pro-angiogenic and proinvasive myofibroblasts," Oncotarget, vol. 6, no. 2, pp. 715731, 2015.

[17] D. Lucchetti, F. Calapà, V. Palmieri et al., "Differentiation affects the release of exosomes from colon cancer cells and their ability to modulate the behavior of recipient cells," The American Journal of Pathology, vol. 187, no. 7, pp. 1633-1647, 2017.

[18] N. Syn, L. Wang, G. Sethi, J.-P. Thiery, and B.-C. Goh, "Exosome-mediated metastasis: from epithelial-mesenchymal transition to escape from immunosurveillance," Trends in Pharmacological Sciences, vol. 37, no. 7, pp. 606-617, 2016.

[19] M. Gong, B. Yu, J. Wang et al., "Mesenchymal stem cells release exosomes that transfer miRNAs to endothelial cells and promote angiogenesis," Oncotarget, vol. 8, no. 28, pp. 45200-45212, 2017.

[20] J. Sadri Nahand, M. Moghoofei, A. Salmaninejad et al., "Pathogenic role of exosomes and microRNAs in HPV-mediated inflammation and cervical cancer: a review," International Journal of Cancer, vol. 146, no. 2, pp. 305-320, 2020.

[21] J. Hamblin, F. Bokharaei-Salim, M. Karimzadeh et al., "MicroRNAs and exosomes: key players in HIV pathogenesis," HIV Medicine, vol. 21, no. 4, pp. 246-278, 2019.

[22] C. Chiozzini, C. Arenaccio, E. Olivetta et al., "Trans-dissemination of exosomes from HIV-1-infected cells fosters both HIV-1 trans-infection in resting CD4+ T lymphocytes and reactivation of the HIV-1 reservoir," Archives of Virology, vol. 162, no. 9, pp. 2565-2577, 2017.

[23] Y. Fu, L. Zhang, F. Zhang et al., "Exosome-mediated miR146a transfer suppresses type I interferon response and facilitates EV71 infection," PLoS Pathogens, vol. 13, no. 9, Article ID e1006611, 2017.

[24] S. B. Ye, Z. L. Li, D. H. Luo et al., "Tumor-derived exosomes promote tumor progression and T-cell dysfunction through the regulation of enriched exosomal microRNAs in human nasopharyngeal carcinoma," Oncotarget, vol. 5, no. 14, pp. 5439-5452, 2014.

[25] N. Kosaka, H. Iguchi, K. Hagiwara, Y. Yoshioka, F. Takeshita, and T. Ochiya, "Neutral sphingomyelinase 2 (nSMase2)-dependent exosomal transfer of angiogenic microRNAs regulate cancer cell metastasis," Journal of Biological Chemistry, vol. 288, no. 15, pp. 10849-10859, 2013.

[26] Y.-L. Hsu, J.-Y. Hung, W.-A. Chang et al., "Hypoxic lung cancer-secreted exosomal miR-23a increased angiogenesis and vascular permeability by targeting prolyl hydroxylase and tight junction protein ZO-1," Oncogene, vol. 36, no. 34, pp. 4929-4942, 2017. 
[27] A. Blomme, K. Fahmy, O. Peulen et al., "Myoferlin is a novel exosomal protein and functional regulator of cancer-derived exosomes," Oncotarget, vol. 7, no. 50, pp. 83669-83683, 2016.

[28] H. C. Christianson, K. J. Svensson, T. H. van Kuppevelt, J.-P. Li, and M. Belting, "Cancer cell exosomes depend on cellsurface heparan sulfate proteoglycans for their internalization and functional activity," Proceedings of the National Academy of Sciences, vol. 110, no. 43, pp. 17380-17385, 2013.

[29] H. Zhao, Q. Shang, Z. Pan et al., "Exosomes from adiposederived stem cells attenuate adipose inflammation and obesity through polarizing M2 macrophages and beiging in white adipose tissue," Diabetes, vol. 67, no. 2, pp. 235-247, 2018.

[30] Y. Song, H. Dou, X. Li et al., "Exosomal miR-146a contributes to the enhanced therapeutic efficacy of interleukin- $1 \beta$-primed mesenchymal stem cells against sepsis," Stem Cells, vol. 35, no. 5, pp. $1208-1221,2017$.

[31] F. Xu, J.-Y. Zhong, X. Lin et al., "Melatonin alleviates vascular calcification and ageing through exosomal miR-204/miR-211 cluster in a paracrine manner," Journal of Pineal Research, vol. 68, no. 3, p. e12631, 2020.

[32] M. Hou, J. Yang, X. Zhao et al., "Circulating myocardial microRNAs from infarcted hearts are carried in exosomes and mobilise bone marrow progenitor cells," Nature Communications, vol. 10, no. 1, p. 959, 2019.

[33] M. R. Haymart, N. H. Esfandiari, M. T. Stang, and J. A. Sosa, "Controversies in the management of low-risk differentiated thyroid cancer," Endocrine Reviews, vol. 38, no. 4, pp. 351-378, 2017.

[34] D. F. Schneider and H. Chen, "New developments in the diagnosis and treatment of thyroid cancer," CA: A Cancer Journal for Clinicians, vol. 63, no. 6, pp. 374-394, 2013.

[35] S. McGuire, "World cancer report 2014," World Health Organization, International Agency for Research on Cancer, WHO Press, Geneva, Switzerland, 2015.

[36] H. Hardin, H. Helein, K. Meyer et al., "Thyroid cancer stemlike cell exosomes: regulation of EMT via transfer of lncRNAs," Laboratory Investigation, vol. 98, no. 9, pp. 1133-1142, 2018.

[37] R. Ranganath, M. A. Shah, and A. R. Shah, "Anaplastic thyroid cancer," Current Opinion in Endocrinology \& Diabetes and Obesity, vol. 22, no. 5, pp. 387-391, 2015.

[38] A. Maniakas, L. Davies, and M. E. Zafereo, "Thyroid disease around the world," Otolaryngologic Clinics of North America, vol. 51, no. 3, pp. 631-642, 2018.

[39] J. J. Smith, X. Chen, D. F. Schneider et al., "Cancer after thyroidectomy: a multi-institutional experience with 1,523 patients," Journal of the American College of Surgeons, vol. 216, no. 4, pp. 571-577, 2013.

[40] M. Xing, "Molecular pathogenesis and mechanisms of thyroid cancer," Nature Reviews Cancer, vol. 13, no. 3, pp. 184-199, 2013.

[41] J. C. Lee, J. T. Zhao, R. J. Clifton-Bligh et al., "MicroRNA-222 and microRNA-146b are tissue and circulating biomarkers of recurrent papillary thyroid cancer," Cancer, vol. 119, no. 24, pp. 4358-4365, 2013.

[42] R. Sidhu, V. Burdakov, T. Shtam et al., "Plasma exosomal miR-21 and miR-181a differentiates follicular from papillary thyroid cancer," Tumor Biology, vol. 37, no. 9, pp. 12011-12021, 2016.

[43] C. Kolesnikov, Y. Wei, L. Yu, and Y. Xiao, "Identification of altered circular RNA expression in serum exosomes from patients with papillary thyroid carcinoma by high-throughput sequencing," Medical Science Monitor, vol. 25, pp. 2785-2791, 2019.
[44] Z. Wang, J. Lv, X. Zou et al., "A three plasma microRNA signature for papillary thyroid carcinoma diagnosis in Chinese patients," Gene, vol. 693, pp. 37-45, 2019.

[45] N. Edo, K. Kawakami, Y. Fujita et al., "Exosomes expressing thyrotropin receptor attenuate autoantibody-mediated stimulation of cyclic adenosine monophosphate production," Thyroid, vol. 29, no. 7, pp. 1012-1017, 2019.

[46] D. Luo, S. Zhan, W. Xia, L. Huang, W. Ge, and T. Wang, "Proteomics study of serum exosomes from papillary thyroid cancer patients," Endocrine-related Cancer, vol. 25, no. 10, pp. 879-891, 2018.

[47] F. Wu, F. Li, X. Lin et al., "Exosomes increased angiogenesis in papillary thyroid cancer microenvironment," Endocrine-related Cancer, vol. 26, no. 5, pp. 525-538, 2019.

[48] W. Ye, X. Deng, and Y. Fan, "Exosomal miRNA423-5p mediated oncogene activity in papillary thyroid carcinoma: a potential diagnostic and biological target for cancer therapy," Neoplasma, vol. 66, no. 4, pp. 516-523, 2019.

[49] R. G. Hardy, C. Vicente-Dueñas, I. González-Herrero et al., "Snail family transcription factors are implicated in thyroid carcinogenesis," The American Journal of Pathology, vol. 171, no. 3, pp. 1037-1046, 2007.

[50] J. Massagué and A. C. Obenauf, "Metastatic colonization by circulating tumour cells," Nature, vol. 529, no. 7586, pp. 298-306, 2016.

[51] J. P. Thiery, "Epithelial-mesenchymal transitions in tumour progression," Nature Reviews Cancer, vol. 2, no. 6, pp. 442454, 2002.

[52] M. Boufraqech, L. Zhang, M. Jain et al., "miR-145 suppresses thyroid cancer growth and metastasis and targets AKT3," Endocrine-related Cancer, vol. 21, no. 4, pp. 517-531, 2014.

[53] E. Mianehsaz, H. R. Mirzaei, M. Mahjoubin-Tehran et al., "Mesenchymal stem cell-derived exosomes: a new therapeutic approach to osteoarthritis?" Stem Cell Research \& Therapy, vol. 10, no. 1, p. 340, 2019.

[54] Y.-L. Tai, K.-C. Chen, J.-T. Hsieh, and T.-L. Shen, "Exosomes in cancer development and clinical applications," Cancer Science, vol. 109, no. 8, pp. 2364-2374, 2018.

[55] A. Mokhtari, B. Costa-Silva, T.-L. Shen et al., "Tumour exosome integrins determine organotropic metastasis," $\mathrm{Na}$ ture, vol. 527, no. 7578, pp. 329-335, 2015.

[56] K. E. Richards, A. E. Zeleniak, M. L. Fishel, J. Wu, L. E. Littlepage, and R. Hill, "Cancer-associated fibroblast exosomes regulate survival and proliferation of pancreatic cancer cells," Oncogene, vol. 36, no. 13, pp. 1770-1778, 2017.

[57] H. Qi, C. Liu, L. Long et al., "Blood exosomes endowed with magnetic and targeting properties for cancer therapy," ACS Nano, vol. 10, no. 3, pp. 3323-3333, 2016.

[58] L. Hou, U. Erb, K. Zhao, T. Hackert, and M. Zoller, "Efficacy of vaccination with tumor-exosome loaded dendritic cells combined with cytotoxic drug treatment in pancreatic cancer," Oncoimmunology, vol. 6, no. 6, Article ID e1319044, 2017.

[59] F. Khatami and S. M. Tavangar, "Liquid biopsy in thyroid cancer: new insight," International Journal of HematologyOncology and Stem Cell Research, vol. 12, no. 12, pp. 235-248, 2018.

[60] M. Celano, F. Rosignolo, V. Maggisano et al., "MicroRNAs as biomarkers in thyroid carcinoma," International Journal of Genomics, vol. 2017, Article ID 6496570, 11 pages, 2017.

[61] D. V. P. Araque, A. Bleyer, and J. P. Brito, "Thyroid cancer in adolescents and young adults," Future Oncology, vol. 13, no. 14, pp. 1253-1261, 2017. 
[62] M. E. Cabanillas, D. G. McFadden, and C. Durante, "Thyroid cancer," The Lancet, vol. 388, no. 10061, pp. 2783-2795, 2016.

[63] B. R. Roman, L. G. Morris, and L. Davies, "The thyroid cancer epidemic, 2017 perspective," Current Opinion in Endocrinology \& Diabetes and Obesity, vol. 24, no. 5, pp. 332-336, 2017.

[64] T. G. Pemayun, "Current diagnosis and management of thyroid nodules," Acta Medica Indonesiana, vol. 48, no. 48, pp. 247-257, 2016.

[65] B. R. Haugen, "2015 American thyroid association management guidelines for adult patients with thyroid nodules and differentiated thyroid cancer: what is new and what has changed?" Cancer, vol. 123, no. 3, pp. 372-381, 2017.

[66] J. P. Brito, L. Davies, C. Zeballos-Palacios, J. C. Morris, and V. M. Montori, "Papillary lesions of indolent course: reducing the overdiagnosis of indolent papillary thyroid cancer and unnecessary treatment," Future Oncology, vol. 10, no. 1, pp. 1-4, 2014.

[67] G. Rappa, C. Puglisi, M. F. Santos, S. Forte, L. Memeo, and A. Lorico, "Extracellular vesicles from thyroid carcinoma: the new frontier of liquid biopsy," International Journal of Molecular Sciences, vol. 20, no. 5, 2019.

[68] Y. Zhang, W. Zhao, Y. Zhao et al., "Comparative glycoproteomic profiling of human body fluid between healthy controls and patients with papillary thyroid carcinoma," Journal of Proteome Research, 2019.

[69] P. Lin, Z. Yao, Y. Sun et al., "Deciphering novel biomarkers of lymph node metastasis of thyroid papillary microcarcinoma using proteomic analysis of ultrasound-guided fine-needle aspiration biopsy samples," Journal of Proteomics, vol. 204, Article ID 103414, 2019.

[70] K. Pazaitou-Panayiotou, G. Panagiotou, S. A. Polyzos, and C. S. Mantzoros, "Serum adiponectin and insulin-like growth factor 1 IN predominantly female patients with thyroid cancer: association with the histologic characteristics OF the tumor," Endocrine Practice, vol. 22, no. 1, pp. 68-75, 2016.

[71] F. Rosignolo, M. Sponziello, L. Giacomelli et al., "Identification of thyroid-associated serum microRNA profiles and their potential use in thyroid cancer follow-up," Journal of the Endocrine Society, vol. 1, no. 1, pp. 3-13, 2017.

[72] S. Cantara, T. Pilli, G. Sebastiani et al., "Circulating miRNA95 and miRNA190 are sensitive markers for the differential diagnosis of thyroid nodules in a Caucasian population," The Journal of Clinical Endocrinology \& Metabolism, vol. 99, no. 11, pp. 4190-4198, 2014.

[73] M. Li, Q. Song, H. Li, Y. Lou, and L. Wang, "Circulating miR25-3p and miR-451a may Be potential biomarkers for the diagnosis of papillary thyroid carcinoma," PLoS One, vol. 10, no. 7, Article ID e0132403, 2015.

[74] F. Rosignolo, L. Memeo, F. Monzani et al., "MicroRNA-based molecular classification of papillary thyroid carcinoma," International Journal of Oncology, vol. 50, no. 5, pp. 1767-1777, 2017.

[75] S. Martinetti, I. A. Batista, J. L. Costa, and S. A. Melo, "Liquid biopsy beyond circulating tumor cells and cell-free DNA," Acta Cytologica, vol. 63, no. 6, pp. 479-488, 2019.

[76] C. Yang, H. S. Kim, and G. Song, "The potential role of exosomes derived from ovarian cancer cells for diagnostic and therapeutic approaches," Journal of Cellular Physiology, vol. 234, no. 12, pp. 21493-21503, 2019.

[77] E. Campos-Fernandez, L. S. Barcelos, A. G. de Souza, L. R. Goulart, and V. Alonso-Goulart, "Research landscape of liquid biopsies in prostate cancer," American Journal of Cancer Research, vol. 9, no. 7, pp. 1309-1328, 2019.
[78] T. Huang, C. Song, L. Zheng, L. Xia, Y. Li, and Y. Zhou, "The roles of extracellular vesicles in gastric cancer development, microenvironment, anti-cancer drug resistance, and therapy," Molecular Cancer, vol. 18, no. 1, p. 62, 2019.

[79] A. Hesari, S. A. Golrokh Moghadam, A. Siasi et al., "Tumorderived exosomes: potential biomarker or therapeutic target in breast cancer?" Journal of Cellular Biochemistry, vol. 119, no. 6, pp. 4236-4240, 2018.

[80] G. M. Shankar, L. Balaj, S. L. Stott, B. Nahed, and B. S. Carter, "Liquid biopsy for brain tumors," Expert Review of Molecular Diagnostics, vol. 17, no. 10, pp. 943-947, 2017.

[81] J. C. Lee, J.-T. Zhao, J. Gundara, J. Serpell, L. A. Bach, and S. Sidhu, "Papillary thyroid cancer-derived exosomes contain miRNA-146b and miRNA-222," Journal of Surgical Research, vol. 196, no. 1, pp. 39-48, 2015.

[82] K. Prasek, M. T. Płazińska, and L. Królicki, “Diagnosis and treatment of Graves' disease with particular emphasis on appropriate techniques in nuclear medicine. General state of knowledge," Nuclear Medicine Review, vol. 18, no. 2, pp. 110-116, 2015.

[83] T. J. Smith and L. Hegedüs, "Graves' disease," New England Journal of Medicine, vol. 375, no. 16, pp. 1552-1565, 2016.

[84] B. McIver and J. C. Morris, "The pathogenesis of Graves' disease," Endocrinology and Metabolism Clinics of North America, vol. 27, no. 1, pp. 73-89, 1998.

[85] O. Alsanea and O. H. Clark, "Treatment of Graves' disease: the advantages of surgery," Endocrinology and Metabolism Clinics of North America, vol. 29, no. 2, pp. 321-337, 2000.

[86] I. Hiratsuka, H. Yamada, E. Munetsuna, S. Hashimoto, and M. Itoh, "Circulating MicroRNAs in Graves' disease in relation to clinical activity," Thyroid, vol. 26, no. 10, pp. 1431-1440, 2016.

[87] M. Rossi, A. R. Taddei, I. Fasciani, R. Maggio, and F. Giorgi, "The cell biology of the thyroid-disrupting mechanism of dichlorodiphenyltrichloroethane (DDT)," Journal of Endocrinological Investigation, vol. 41, no. 1, pp. 67-73, 2018. 